\title{
A DIVISOR PROBLEM FOR POLYNOMIALS
}

\author{
BENJAMIN KLAHN
}

\begin{abstract}
We characterize all monic polynomials $f(x) \in \mathbb{Z}[x]$ that have the property that

$f(p) \mid f\left(p^{p}\right)$, for all sufficiently large primes $p \geq N(f)$.

We also give necessary conditions and a sufficient condition for monic polynomials $f(x) \in \mathbb{Z}[x]$ to satisfy $f(p) \mid f\left(p^{p}\right)$ for all primes $p$.
\end{abstract}

\section{INTRODUCTION}

For integer polynomials $f(x)$ it is a very difficult problem to say something useful about the size and the number of prime divisors of values $f(n)$, $n \in \mathbb{Z}$. It is conjectured that irreducible integer polynomials $f(x)$ without any fixed prime divisor take prime values for infinitely many $n$, and it is even believed that there are infinitely many prime numbers $p$ for which $f(p)$ is prime. This is a well-studied problem and there are deep results towards this conjecture. In [ID82 Deshouillers and Iwaniec proved that there are infinitely many $n$ such that $n^{2}+1$ has a prime divisor larger than $n^{6 / 5}$. In [HB00] Heath-Brown proved that for an explicitly given positive $\delta$ the largest prime divisor of $n^{3}+2$ is larger than $X^{1+\delta}$ for a positive proportion of integers $n$ in the interval $[X, 2 X]$. Later Irving improved this result in [115] by showing that one can take $\delta=10^{-52}$. However, it is not known for a single primitive irreducible polynomial of degree at least 2 whether it takes infinitely many prime values.

On the other hand, in [B20] it was shown by Bober, Fretwell, Martin and Wooley that for a fixed quadratic integer polynomial $f(x)$ and a positive $\epsilon$ there are infinitely many integers $n$ such that all prime divisors of $f(n)$ are less than $n^{\epsilon}$.

Thus, it is typically a very difficult problem obtaining information about the prime divsiors of elements of a sequence $(f(n))_{n=m}^{M}$, and initially it therefore came as a surprise to the author that it was possible to relate the prime divsisors of $f(p)$ and $f\left(p^{p}\right)$.

2010 Mathematics Subject Classification. 11A07; 11C08; $11 \mathrm{~T} 06$.

Key words and phrases. Integer polynomials, divsibility of polynomial values, prime numbers. 
However, the density theorem of Chebotarëv often comes in very handy as it gives complete information on the density of primes which divide some value of $f(x)$ in terms of its Galois group $\operatorname{Gal}(\operatorname{split}(f(x), \mathbb{Q}) / \mathbb{Q})$ where $\operatorname{split}(f(x), \mathbb{Q})$ is the splitting field of $f(x)$. In fact, Chebotarëv's density theorem is one of the key tools in our study of which monic integer polynomials, $f(x)$, satisfy the condition

$$
f(p) \mid f\left(p^{p}\right) \text { for all sufficiently large primes } p \geq N(f) .
$$

Related to the problem of determining the polynomials that satisfy (1.1) is the following problem.

Find all integer polynomials $f(x)$ with nonnegative coefficients such that

$$
\operatorname{rad}(f(n)) \mid f\left(n^{\operatorname{rad}(n)}\right)
$$

for all nonnegative integers $n$.

Here the radical, $\operatorname{rad}(\cdot)$, is defined in the following way.

Definition 1.1. For a nonnegative integer $n$ define $\operatorname{rad}(n)=1$ if $n=0$ or $n=1$, and otherwise $\operatorname{rad}(n)=p_{1} p_{2} \cdots p_{k}$ where $p_{1}<p_{2}<\cdots<p_{k}$ are all the distinct prime factors of $n$.

The problem above was submitted for the International Mathematical Olympiad in 20121, see [IMO12]. Altering the formulation of the olympiad problem to only having information at primes makes the problem harder, and the answer to the problem of which monic integer polynomials satisfy (1.1) is richer in the sense that there are more types of polynomials satisfying this condition than the condition of the olympiad problem.

\section{Results}

The main result in this paper is Theorem 2.1 which gives a necessary and sufficient condition for (1.1) to be satisfied.

Theorem 2.1. Let $f(x) \in \mathbb{Z}[x]$ be a monic polynomial. There is a positive integer $N:=N(f)$ such that

$$
f(p) \mid f\left(p^{p}\right), \text { for all primes } p \geq N,
$$

if and only if there are positive integers $d_{1}, \ldots, d_{m}$ and nonnegative integers $e_{0}, e_{1}, \ldots, e_{m}$ such that

$$
f(x)=x^{e_{0}} \Phi_{d_{1}}(x)^{e_{1}} \Phi_{d_{2}}(x)^{e_{2}} \cdots \Phi_{d_{m}}(x)^{e_{m}},
$$

\footnotetext{
${ }^{1}$ A polynomial, $f(x)$, satisfies this condition if and only if $f(x)=a x^{m}$ for some nonnegative integers $a$ and $m$
} 
where $\Phi_{d}(x)$ is the d-th cyclotomic polynomial.

Typically a polynomial of the form (2.2) will not satisfy (2.1) with $N=2$. Of course there are the trivial solutions $f(x)=x^{m}$ for an arbitrary positive integer $m$, that satisfy (2.1) with $N=2$. Furthermore, all polynomials of the form $f(x)=x^{m}-1$ for an arbitrary positive integer $m$, satisfy (2.1) with $N=2$. A less trivial example of such a polynomial is $f(x)=$ $(x-1)(x+1)\left(x^{2}+x+1\right)$. It turns out that a polynomial that satisfies (2.1) with $N=2$ and is not a power of $x$ has 1 as a root.

Theorem 2.2. Let $f(x) \in \mathbb{Z}[x]$ be a monic polynomial that satisfies (2.1) with $N=2$. If $f(x)$ is not a power of $x$ then $x-1=\Phi_{1}(x) \mid f(x)$. Furthermore, a polynomial of the form (2.2) with $d_{1}=1$ and $d_{j}=p_{j}$ for $j \geq 2$ for distinct primes $p_{j}$ satisfies (2.1) with $N=2$.

\section{PRELiminaries}

Along the way to deriving Theorem 2.1 we will make use of the following consequence of the Chebotarëv density theorem.

Theorem 3.1 ([SL95]). Let $f(x) \in \mathbb{Z}[x]$ be a monic polynomial having nonzero discriminant $\Delta(f(x)) \neq 0$. For a positive proportion of the primes, the reduction $f(x)$ mod $p \in \mathbb{F}_{p}[x]$ will split completely as the product of distinct linear factors.

Theorem 3.1 will be crucial because it will allow us to apply the following trick that later will show that any solution $f(x)$ must, essentially, be a product of cyclotomic polynomomials.

Lemma 3.2. Let $g(x)$ and $h(x)$ be monic integer polynomials with $h(x)$ having discriminant $\Delta(h(x)) \neq 0$. Assume that for infinitely many primes $p$, for which $h(x)$ mod $p$ splits completely, $g(x)$ has the following property:

$$
k \in \mathbb{Z} \text { and } h(k) \equiv 0 \quad(\bmod p) \Longrightarrow g(k) \equiv 0 \quad(\bmod p) .
$$

Then $h(x)$ divides $g(x)$ in $\mathbb{Z}[x]$.

Remark 3.3. In relation to Lemma 3.2 we note that for monic integer polynomials $g(x)$ and $h(x)$ satisfying the conditions in Lemma 3.2 it holds that for infinitely many primes $p,(h(x) \bmod p) \mid(g(x) \bmod p)$. As such, Lemma 3.2 can be generalized since the existence of infinitely many primes $p$ such that $(h(x) \bmod p) \mid(g(x) \bmod p)$, in fact, implies that $h(x)$ divides $g(x)$ in $\mathbb{Z}[x]$ - regardless of $h(x)$ having nonzero discriminant. However, for later convienience we prefer to use the formulation of Lemma 3.2 as we believe it will make the proof of Theorem 2.1 more clear. 
We give the proof of Lemma 3.2 in the next section.

In order to show that all polynomials of the form (2.2) satisfy (2.1) for all large primes we will use the following two elementary properties of cyclotomic polynomials.

Lemma 3.4. For cyclotomic polynomials the following holds:

(1) For a prime $p$ and a positive integer $d$, we have

$$
\Phi_{d}\left(x^{p}\right)= \begin{cases}\Phi_{p d}(x) & \text { if } p \mid d \\ \Phi_{p d}(x) \Phi_{d}(x) & \text { if } p \nmid d .\end{cases}
$$

(2) If $p$ is a prime and $n$ and $m$ are positive integers such that

$$
p \mid\left(\Phi_{n}(b), \Phi_{m}(b)\right)
$$

for some integer $b \geq 2$, then $m / n=p^{a}$ for some integer $a$.

The first property is Lemma 3 in D12 and the second property easily follows from Lemma 2 in [F02].

Definition 3.5. A primitive prime divisor $p$ for a term $a_{n}$ in an integer sequence $\left(a_{i}\right)_{i=1}^{\infty}$ is a prime dividing $a_{n}$ but not dividing $a_{i}$ for all positive integers $i<n$.

Lemma 3.6 (Zsigmondy's theorem, EW05]). Let $b \geq 2$ be an integer. Any term in the sequence $\left(\Phi_{d}(b)\right)_{d=1}^{\infty}$ has a primitive prime divisor, except for the cases

- $b=2$ and $d=1$,

- $b=2^{a}-1$ and $d=2$,

- $b=2$ and $d=6$.

We will show that $\Phi_{d}(b)$ typically has a prime divisor $>d$.

Lemma 3.7. Let $b \geq 2$ and $d$ be positive integers such that $(b, d) \neq(2,1)$, $(b, d) \neq(2,6)$ and $(b, d) \neq\left(2^{a}-1,2\right)$ for all positive integers $a$. Then there is a prime, $p>d$, satisfying $p \mid \Phi_{d}(b)$.

We give the proof of Lemma 3.7 in the next section. The proof in fact implies more, namely that, for $(b, d)$ as in Lemma 3.7. we have that every primitive prime divisor of $\Phi_{d}(b)$ for the sequence $\left(\Phi_{d}(b)\right)_{d=1}^{\infty}$ is greater than $d$.

\section{Proofs of Lemmas 3.2 And 3.7}

Before we prove Theorem 2.1 we will settle Lemma 3.2 and Lemma 3.7. 
Proof of Lemma 3.2. Since the polynomials in question are monic, we can do division in $\mathbb{Z}[x]$ and obtain

$$
g(x)=q(x) h(x)+r(x)
$$

with $q(x), r(x) \in \mathbb{Z}[x]$ and $\operatorname{deg}(r(x))<\operatorname{deg}(h(x))$. We can pick a prime $p$ greater than all coefficients in $r(x)$ such that $h(x) \bmod p$ splits completely in $\mathbb{F}_{p}[x]$ and such that (3.1) is satisfied. Evaluating (4.1) in the $\operatorname{deg}(h(x))$ many distinct roots of $h(x) \bmod p$ implies that $r(x) \bmod p$ must be the zero polynomial. But as $p$ was chosen large this implies that $r(x)=0$ holds in $\mathbb{Z}[x]$. Thus, $h(x)$ divides $g(x)$ in $\mathbb{Z}[x]$ as claimed.

Proof of Lemma 3.7. For $d=1$ the claim is obviously fulfilled. Assume therefore that $d>1$. By Lemma 3.6 we may pick a prime $p$ such that $p \mid \Phi_{d}(b)$ and $p \nmid \Phi_{i}(b)$ for $1 \leq i \leq d-1$. We claim that $p>d$. Assume for the sake of a contradiction that $p \leq d$.

As $\Phi_{d}(0)=1$ we must have $(p, b)=1$, and therefore by Fermat's Little Theorem

$$
p \mid\left(b^{p-1}-1\right)=\prod_{k \mid(p-1)} \Phi_{k}(b),
$$

implying that $p \mid \Phi_{k}(b)$ for some $k \leq p-1<d$, contradicting the fact that $p$ is a primitive prime divisor.

\section{Proof OF THE MAIN RESUlts}

Proof of Theorem [2.1. Let first $f(x) \in \mathbb{Z}[x]$ be such that there is a constant $N=N(f)$ for which (2.1) is satisfied.

As $f(x)$ is monic we can factorize it as, say,

$$
f(x)=x^{a_{0}} p_{1}(x)^{a_{1}} p_{2}(x)^{a_{2}} \cdots p_{m}(x)^{a_{m}}
$$

where the $p_{i}(x)$ are distinct irreducible monic polynomials in $\mathbb{Z}[x]$ different from $x$. Let

$$
g(x):=p_{1}(x) p_{2}(x) \cdots p_{m}(x):=x^{n}+a_{n-1} x^{n-1}+\cdots+a_{1} x+a_{0} .
$$

It is easy to see that $g(x)$ must then also be monic, have a nonzero discriminant and satisfy the weaker condition

$$
\operatorname{rad}(g(p)) \mid g\left(p^{p}\right) \text { for all } p \geq \max \left\{N,\left|a_{0}\right|\right\} .
$$

If $g(x)=1$ we are done, so assume that $\operatorname{deg}(g(x)) \geq 1$.

Lemma 5.1. There is a constant $C:=C(g(x))$ only dependent on $g(x)$ such that there are infinitely many primes $q$ for which $g(x) \bmod q$ splits completely and such that any root of $g(x) \bmod q$ has order at most $C$ modulo $q$. 
Proof. Consider primes $q$ satisfying $q>\max \left\{N,\left|a_{0}\right|\right\}$ and for which there is an integer $r \in \mathbb{Z}$ such that $q \mid g(r)$. Note that as $a_{0} \neq 0$ we must have $(r, q)=$ 1 , and thus by Dirichlet's Theorem for Primes in Arithmetic Progressions there are infinitely many primes $p>r$ such that $p \equiv r(\bmod q)$. Since $p>q>N$, we obtain from (5.1) that

$$
q|\operatorname{rad}(g(p))| g\left(p^{p}\right) .
$$

Thus, $q \mid g\left(r^{p}\right)$ for any prime $p>r$ such that $p \equiv r(\bmod q)$. However, combining the Chinese Remainder Theorem and Dirichlet's Theorem for Primes in Arithmetic Progressions we are free to choose any reduced residue for $p(\bmod q-1)$. Thus, for any positive integer $l$ coprime to $q-1$ we must have $q \mid g\left(r^{l}\right)$. But as the reduction of $g(x)$ modulo $q$ is nonzero there can be at most $\operatorname{deg}(g(x))$ distinct values in the sequence $\left(r^{l} \bmod q\right)_{(l, q-1)=1}$.

By Theorem [3.1, there is a density $\delta \in(0,1]$ of primes $q$ for which $g(x)$ splits completely modulo $q$. Choose $T$ so large that the $T$ first primes $p_{1}, p_{2}, \ldots, p_{T}$ satisfy

$$
\left(p_{1}-1\right)\left(p_{2}-1\right) \cdots\left(p_{T}-1\right) \geq 2 \delta^{-1},
$$

and put $C=p_{T}^{\operatorname{deg}(g(x))+2}$. By the Prime Number Theorem for Primes in Arithmetic Progressions there are infinitely many primes $q$ such that $g(x)$ splits completely modulo $q$ and such that $q \not \equiv 1\left(\bmod p_{1} p_{2} \cdots p_{T}\right)$. By the pigeonhole principle there must be an $1 \leq i \leq T$ such that there are infinitely many such primes $q \not \equiv 1\left(\bmod p_{i}\right)$. Let

$$
Q_{i}:=\left\{q \text { prime }: q \not \equiv 1 \quad\left(\bmod p_{i}\right) \text {, and } g(x) \text { splits completely modulo } q\right\}
$$

which then is an infinite set. We claim that with our choice of $C$ the elements of $Q_{i}$ satisfy that the order of any root of $g(x)$ modulo $q$ is at most $C$.

To check this, let $q \in Q_{i}$ and let $r$ be a root of $g(x)$ modulo $q$. If the order of $r$ modulo $q$ were greater than $C$ we would have

$$
\left\{r^{p_{i}} \bmod q, r^{p_{i}^{2}} \bmod q, \ldots, r^{p_{i}^{\operatorname{deg}(g(x))+1}} \bmod q\right\} \subseteq\left(r^{l} \bmod q\right)_{(l, q-1)=1}
$$

which would be a contradiction as the elements on the left-hand side would be pairwise distinct.

Lemma 5.2. There is an integer $M$ such that $g(x) \mid\left(x^{M}-1\right)$.

Proof. Let $M$ be the least common multiple of all positive integers less than or equal to $C$. By Lemma 5.1 there are then infinitely many primes $q$ for which $g(x)$ modulo $q$ splits completely and any root, $r$, of $g(x) \bmod q$ satisfies $r^{M}-1 \equiv 0(\bmod q)$. Thus, we are in a situation where we can apply Lemma 3.2 to obtain that $g(x) \mid\left(x^{M}-1\right)$ in $\mathbb{Z}[x]$. 
Using the identity

$$
x^{M}-1=\prod_{d \mid M} \Phi_{d}(x)
$$

we now easily obtain that $g(x)$ is the product of certain cyclotomic polynomials and we thereby get that $f(x)$ has the form of (2.2), as wanted.

Now to see that all polynomials of the form (2.2) satisfy (2.1) it clearly suffices to check that for every $i=1,2, \ldots, m$,

$$
\Phi_{d_{i}}(p) \mid \Phi_{d_{i}}\left(p^{p}\right), \text { for all primes } p \geq \max \left\{d_{1}, d_{2}, \ldots, d_{m}\right\}+1 \text {. }
$$

This, however, follows directly from Lemma [3.4, concluding the proof of Theorem 2.1.

Proof of Theorem [2.2. We start by showing that any monic polynomial $f(x) \in \mathbb{Z}[x]$ that satisfies (2.1) with $N=2$, and that is not a power of $x$, must be divisible by $x-1$.

Let

$$
f(x)=x^{e_{0}} \Phi_{d_{1}}(x)^{e_{1}} \Phi_{d_{2}}(x)^{e_{2}} \cdots \Phi_{d_{m}}(x)^{e_{m}}
$$

satisfy (2.1) with $N=2$ and with $f(x)$ not a power of $x$. As $\left(\Phi_{d}(n), n\right)=1$ for all $d$ and $n$ we must also have that $h(x):=f(x) / x^{e_{0}}$ satisfies (2.1) with $N=2$.

Let $\mathcal{A}=\left\{d_{1}, d_{2}, \ldots, d_{m}\right\}$. We then have to show that $1 \in \mathcal{A}$. Let

$$
M:=\operatorname{lcm}\left(d_{1}, d_{2}, \ldots, d_{m}\right):=p_{1}^{a_{1}} p_{2}^{a_{2}} \cdots p_{k}^{a_{k}}
$$

where

$$
p_{1}<p_{2}<\cdots<p_{k}
$$

Lemma 5.3. Let $d \in \mathcal{A}$ for which $p_{k} \mid d$. If $p_{i} \mid d$ and $\left(p_{i}, d\right) \neq(2,6)$ then also $d / p_{i} \in \mathcal{A}$.

Proof. According to Lemma 3.7 $\Phi_{d}\left(p_{i}\right)$ must have a prime factor, $p$, satisfying $p>d \geq p_{k}$. As (2.1), in particular, holds for $p_{i}$ there is an index $j$ such that $p \mid \Phi_{d_{j}}\left(p_{i}^{p_{i}}\right)$. By Lemma 3.4 (1) we have $p \mid \Phi_{d / p_{i}}\left(p_{i}^{p_{i}}\right)$ and Lemma 3.4 (2) then gives that $d_{j} /\left(d / p_{i}\right)$ is a power of $p$. But as $p>p_{k}$ this means that $d_{j}=d / p_{i}$, proving the lemma.

Starting with an element $d \in \mathcal{A}$ that is divisible by $p_{k}$ we may repeatedly apply Lemma 5.3 to peel off prime factors of $d$, in each step replacing $d$ by $d / p_{i}$, where $p_{i}$ is the least prime factor of $d$. Continuing this process as long as possible we obtain either that $1 \in \mathcal{A}$ or that $6 \in \mathcal{A}$ and $p_{k}=3$.

In the first case we are done, so assume that $6 \in \mathcal{A}$ and $p_{k}=3$. As $\Phi_{6}(2)=3$ there must be some $d_{j} \in \mathcal{A}$ with $3 \mid \Phi_{d_{j}}\left(2^{2}\right)$. Since $3 \mid \Phi_{1}(4), d_{j}$ 
must by Lemma 3.4 (2) be a power of 3 . Putting $d=d_{j}:=3^{a}$ we may again repeatedly apply Lemma 5.3, ultimately leading to $1 \in \mathcal{A}$.

For the second statement let $d_{2}=p_{2}, d_{3}=p_{3}, \ldots, d_{m}=p_{m}$ be distinct primes. The first statement in Lemma 3.4 implies that $\Phi_{p_{j}}(p) \mid \Phi_{p_{j}}\left(p^{p}\right)$ for any prime $p \neq p_{j}$. Therefore we only need to worry about $p=p_{j}$ for some $j$. However, $\Phi_{p_{j}}\left(p_{j}\right) \Phi_{1}\left(p_{j}\right)=p_{j}^{p_{j}}-1=\Phi_{1}\left(p_{j}^{p_{j}}\right)$, which settles the case $p=p_{j}$.

Acknowledgements. The author thanks the anonymous referee for providing valuable insights and giving detailed comments. The author also thanks his supervisor, Christian Elsholtz, for commenting on a preprint of the paper. The author acknowledges the support of the Austrian Science Fund (FWF): W1230.

\section{REFERENCES}

[B20] J. W. Bober, D. Fretwell, G. Martin and T. D. Wooley Smooth values of polynomials, Journal of the Australian Mathematical Society 108, no. 2 (2020), 245-261.

[D12] G. Dresden Resultants of Cyclotomic Polynomials, Rocky Mountain Journal Of Mathematics 42, no. 5 (2012), 1461-1469.

[EW05] G. Everest and T. Ward An introduction to number theory, Springer Graduate Texts in Mathematics 232, London: Springer (2005), ISBN: 9781852339173.

[F02] M. Filaseta Coverings of the integers associated with an irreducibility theorem of A. Schinzel, in Number theory for the millennium, II Urbana, IL, 2000, A.K. Peters, Natick, MA, 2002.

[HB00] D. R. Heath-Brown The largest prime factor of $X^{3}+2$, Proc. Lond. Math. Soc. 82 (2000), 554-596.

[I15] A. J. Irving The largest prime factor of $X^{3}+2$, Acta Arithmetica 171 (2015), $67-80$.

[ID82] H. Iwaniec and J. M. Deshouillers, On the greatest prime factor of $n^{2}+1$, Annales-Institut Fourier 32, no. 4 (1982), 1-11.

[IMO12] IMO Shortlist 2012,

URL: https://www .imo-official.org/problems/IM02012SL.pdf

[M84] K. S. McCurley Prime values of polynomials and Irreducibility testing, American Mathematical Society 11, no. 1 (1984), 155-158.

[SL95] P. Stevenhagen and H. W. Lenstra, Jr. Chebotarëv and his density theorem, URL: https://www.math.leidenuniv.nl/ hwl/papers/cheb.pdf

Institute of Analysis And Number Theory, Technical University of Graz, Ḱopernikusgasse 24/II, 8010 Graz, Austria

Email address: klahn@math.tugraz.at 